\title{
On Fuzzy Portfolio Selection Problems: A Parametric Representation Approach
}

\author{
Omid Solaymani Fard and Mohadeseh Ramezanzadeh \\ Department of Applied Mathematics, School of Mathematics and Computer Science, Damghan University, Damghan, Iran \\ Correspondence should be addressed to Omid Solaymani Fard; omidsfard@gmail.com
}

Received 26 May 2017; Accepted 24 July 2017; Published 14 September 2017

Academic Editor: Carla Pinto

Copyright (C) 2017 Omid Solaymani Fard and Mohadeseh Ramezanzadeh. This is an open access article distributed under the Creative Commons Attribution License, which permits unrestricted use, distribution, and reproduction in any medium, provided the original work is properly cited.

\begin{abstract}
Fuzzy portfolio selection problem is a major issue in the financial field and a special case of constrained fuzzy-valued optimization problems (CFOPs). In this respect, the present paper aims to investigate the CFOP with regard to the features of the parametric representation of fuzzy numbers named as convex constraint function (CCF) which is proposed by Chalco-Cano et al. in 2014. Furthermore, relying on this parametric representation, some proper conditions are provided for the existence of solutions to a CFOP. To this end, by the increasing representation of CCF, the main problem is converted to a parametric multiobjective programming problem and some solution concepts from a similar framework in the multiobjective programming are proposed for the CFOP. Eventually to illustrate the proposed results, the fuzzy portfolio selection problem is discussed.
\end{abstract}

\section{Introduction}

In fact, because of using the experimental and empirical data for modeling a real world phenomenon, a deterministic mathematical model may not be a perfectly realistic representation. There are several approaches to deal with such real world phenomena, for example, fuzzy techniques, stochastic models, and interval analysis, which differ by their advantages and disadvantages $[1,2]$. However, in many practical situations, the uncertainties are not of the statistical or interval type; more precisely, this situation happens mainly through the modeling in terms of linguistic expressions that depend on the human judgment. In other words, an expert perceives exactly which values and parameters are possible and which are not. Therefore, the set of all possible values and parameters can be naturally described as fuzzy numbers by the expert's knowledge.

Historically, fuzzy set theory was proposed by Zadeh in [3] and developed considerably by many other researchers. This theory provides conceptually powerful techniques to handle the imperfect information related to vagueness and imprecision.

Nowadays, the fuzzy optimization problem is effective in a lot of different disciplines related to optimization such as operations research engineering, economics, and artificial intelligence [4-7]. It can be said that the fuzzy optimization problem provides an appropriate choice for considering the vagueness and ambiguousness into the formulation and solutions of the multitude of optimization problems. Indeed, there are several motivations to apply fuzzy optimization model; first, it deals with some practical optimization problems more conveniently than conventional optimization model; also, fuzzy optimization model efficiently reduces information loss arising from the traditional optimization model; moreover, it allows the designer to implement linguistic constraints that may not be easily defined using more conventional optimization algorithms; finally, this model may permit managers to have not only one solution but also a set of them, so that the most suitable solution can be applied according to the state of existing decision of the production process at a given time and without increasing delay. Furthermore, accessing a set of solutions enables user to investigate and analyze the system information in more detail.

On the other hand, most of the common portfolio selection models deal with the uncertainty via probabilistic approaches, where those probabilistic approaches only partly 
capture the reality. In addition, there are some other techniques that manage the uncertainty of the financial markets as the fuzzy set theory. It is noteworthy that the fuzzy portfolio selection model integrates the quantitative and qualitative analysis, experts' knowledge, and the investors' opinion in a better manner [7]. Therefore, in this paper, the portfolio selection problem under fuzzy environment based on the constrained fuzzy optimization problem is going to be studied. Many modern computing methodologies can be seen for various fuzzy systems, for example, [8-14]. It is also worth mentioning that there are several results associated with parametric representations of fuzzy numbers [15-17]. Recently, Chalco-Cano et al. [15] have proposed two parametric representations for interval numbers named as "increasing/decreasing convex constraint function" and then explicitly extended the proposed representations to the fuzzy case. The representations have the advantage of allowing flexible and easy-to-control shapes of the fuzzy numbers and it is very simple to implement [17]. Accordingly, this point of view and its increasing parametric representation motivate us here to study the fuzzy portfolio selection problem as an application of the constrained fuzzy-valued optimization problem. To this end, the arithmetic of fuzzy numbers and the calculus of fuzzy-valued functions are developed based on this parametric representation. Then, by parametric representation of fuzzy-valued function, the constrained fuzzy optimization problem is transformed into a deterministic multiobjective problem. Besides, some solution concepts from a similar framework in the multiobjective programming problem are proposed for the constrained fuzzy-valued optimization problem, by converting it to a general constrained optimization problem. Finally, it has been demonstrated that the solution of the general optimization is related to the solution of the main problem.

The rest of the paper is organized as follows. Section 2 is devoted to giving the definitions of fuzzy numbers and some arithmetic that are used later in the development of results in fuzzy environment. The fuzzy-valued functions in the parametric form and their properties, calculus and convexity, are studied in Section 3. In Section 4, the constrained fuzzy-valued optimization problem is discussed and, as an application, the proposed method is considered to the fuzzyvalued quadratic programming problem. In Section 5, two numerical examples are established to confirm the efficacy of the proposed approach; more particularly one of them reveals how to solve the fuzzy portfolio selection problem. At the end, the conclusion is made in Section 6.

\section{Fuzzy Numbers and Their Arithmetic}

In this section, some basic notations and results on the fuzzy arithmetic are presented; however, it is assumed that the reader is familiar with the fuzzy theory.

Definition 1 (see [18]). Let $\tilde{a}: \mathbb{R} \rightarrow[0,1]$ be a fuzzy set on the set of real numbers $\mathbb{R}$. The fuzzy set $\tilde{a}$ is a fuzzy number if it is a normal, convex, upper semicontinuous, and compactly supported.
The set of all fuzzy numbers on $\mathbb{R}$ is denoted by $\mathscr{F}(\mathbb{R})$. For all $\alpha \in(0,1], \alpha$-level set $[\tilde{a}]^{\alpha}$ of any $\tilde{a} \in \mathscr{F}(\mathbb{R})$ is defined as $[\widetilde{a}]^{\alpha}=\{x \in \mathbb{R} ; \tilde{a}(x) \geq \alpha\}$. The 0 -level set $[\widetilde{a}]^{0}$ is defined as the closure of the set $\{x \in \mathbb{R} ; \widetilde{a}(x)>0\}$. By Definition 1 , for any $\tilde{a} \in \mathscr{F}(\mathbb{R})$ and for each $\alpha \in(0,1],[\tilde{a}]^{\alpha}$ is a compact and convex subset of $\mathbb{R}$ and $[\tilde{a}]^{\alpha}=\left[\underline{a}^{\alpha}, \bar{a}^{\alpha}\right] . \tilde{a} \in \mathscr{F}(\mathbb{R})$ can be recovered from its $\alpha$-level by a well-known decomposition theorem, which states that

$$
\widetilde{a}=\bigcup_{\alpha \in[0,1]} \alpha[\widetilde{a}]^{\alpha},
$$

where $\alpha[\tilde{a}]^{\alpha}$ denotes the algebraic product of a scalar $\alpha$ with the $\alpha$-level set $[\widetilde{a}]^{\alpha}$ and union on the right-hand side is the standard fuzzy union.

As previously mentioned, fuzzy numbers and their arithmetic can be expressed in terms of parameters in the several models [15-17]. Here, from increasing parametric representation [15], each $\alpha$-level of an arbitrary fuzzy number $\widetilde{a} \in \mathscr{F}(\mathbb{R})$ is represented alternatively by its bounds as follows:

$$
\begin{aligned}
{[\widetilde{a}]^{\alpha} } & =\left[\underline{a}^{\alpha}, \bar{a}^{\alpha}\right] \\
& =\left\{a(t, \alpha)=\underline{a}^{\alpha}+t\left(\bar{a}^{\alpha}-\underline{a}^{\alpha}\right) \mid t, \alpha \in[0,1]\right\},
\end{aligned}
$$

which is based on the convex combination of upper and lower bounds. Moreover, by the parametric representation (2), the $\alpha$-level of a $k$-dimensional fuzzy vector $\widetilde{\mathbf{C}}_{v}^{k} \in(\mathscr{F}(\mathbb{R}))^{k}$ and a fuzzy matrix $\widetilde{\mathbf{A}}_{m} \in(\mathscr{F}(\mathbb{R}))^{p \times q}$ can be represented as the set of real-valued vectors and matrices, respectively; that is,

$$
\begin{aligned}
& {\left[\widetilde{\mathbf{C}}_{\nu}^{k}\right]^{\alpha}=\left[\left(\widetilde{c}_{1}, \widetilde{c}_{2}, \ldots, \widetilde{c}_{k}\right)^{T}\right]^{\alpha}=\{c(\mathbf{t}, \alpha) \mid c(\mathbf{t}, \alpha)} \\
& \quad=\left(c_{1}\left(t_{1}, \alpha\right), c_{2}\left(t_{2}, \alpha\right), \ldots, c_{k}\left(t_{k}, \alpha\right)\right)^{T}, c_{i}\left(t_{i}, \alpha\right) \\
& \quad=c_{i}^{\alpha}+t_{i}\left(\bar{c}_{i}^{\alpha}-\underline{c}_{i}^{\alpha}\right), \mathbf{t}=\left(t_{1}, \ldots, t_{k}\right)^{T}, 0 \leq t_{i} \leq 1, i \\
& \quad=1, \ldots, k, 0 \leq \alpha \leq 1\}, \\
& {\left[\widetilde{\mathbf{A}}_{m}\right]^{\alpha}=\{A(\mathbf{t}, \alpha) \mid A(\mathbf{t}, \alpha)} \\
& \quad=\left(a_{i j}\left(t_{i j}, \alpha\right)\right)_{p \times q}, a_{i j}\left(t_{i j}, \alpha\right)=\underline{a}_{i j}^{\alpha} \\
& \quad+t_{i j}\left(\bar{a}_{i j}^{\alpha}-\underline{a}_{i j}^{\alpha}\right), 0 \leq t_{i j} \leq 1, i=1,2, \ldots, p, j \\
& \quad=1,2, \ldots, q, 0 \leq \alpha \leq 1\} .
\end{aligned}
$$

The parametric representation (2) helps us to build the fuzzy arithmetic, immediately. Nevertheless, the binary operations between two arbitrary fuzzy number can be defined in terms of parameter as follows.

Definition 2. For $\widetilde{a}, \widetilde{b} \in \mathscr{F}(\mathbb{R})$, the algebraic operations can be defined as

$$
[\tilde{a} \circledast \tilde{b}]^{\alpha}=\left\{a\left(t_{1}, \alpha\right) * b\left(t_{2}, \alpha\right) \mid t_{1}, t_{2}, \alpha \in[0,1]\right\},
$$




$$
\begin{aligned}
& {[\tilde{a} \oslash \widetilde{b}]^{\alpha}} \\
& \quad=\left\{\frac{a\left(t_{1}, \alpha\right)}{b\left(t_{2}, \alpha\right)} \mid t_{1}, t_{2}, \alpha \in[0,1], b\left(t_{2}, \alpha\right) \neq 0\right\}, \\
& {[k \widetilde{a}]^{\alpha}=\{k a(t, \alpha) \mid t, \alpha \in[0,1]\},}
\end{aligned}
$$

where $* \in\{+,-, \cdot\}$ and $k \in \mathbb{R}$.

Remark 3. It is clear that $\widetilde{a} \ominus \widetilde{b} \neq \tilde{a} \ominus_{H} \widetilde{b}$ in general, where $\ominus_{H}$ is the Hukuhara difference. However, it can be deduced that $\widetilde{a} \ominus_{H} \widetilde{b}=\widetilde{a} \ominus \widetilde{b}$ if, in (5), $t=t_{1}=t_{2}$ and $c(t, \alpha)=a(t, \alpha)-b(t, \alpha)$ is a nondecreasing function for all $t, \alpha \in[0,1]$.

Definition 4. The product of $k$-dimensional fuzzy vector $\widetilde{\mathbf{C}}_{v}^{k}=$ $\left(\widetilde{c}_{1}, \widetilde{\mathcal{c}}_{2}, \ldots, \widetilde{\mathcal{c}}_{k}\right)^{T} \in(\mathscr{F}(\mathbb{R}))^{k}$ and a $k$-dimensional real vector $d=\left(d_{1}, d_{2}, \ldots, d_{k}\right)^{T} \in \mathbb{R}^{k}$ is defined as $\left(\widetilde{\mathbf{C}}_{\nu}^{k}\right)^{T} \diamond d=\sum_{j=1}^{k} \widetilde{c}_{j} d_{j}$, where it is a fuzzy number.

It is noteworthy that fuzzy numbers are frequently partial ordered. In fact, there are many ways to define the fuzzy order among the set of all fuzzy numbers [19-22]. For example, Ramík and ímánek [22] proposed a partial order relation called the fuzzy-max order; Molinari [20] considered a new criterion of choice between generalized triangular fuzzy numbers and so on. In this paper, two specific partial ordering relations on fuzzy numbers using parametric representation are introduced.

Definition 5. For two arbitrary $\tilde{a}, \widetilde{b} \in \mathscr{F}(\mathbb{R})$, with parametric representations $[\widetilde{a}]^{\alpha}=\{a(t, \alpha) \mid t, \alpha \in[0,1]\}$ and $[\widetilde{b}]^{\alpha}=$ $\{b(t, \alpha) \mid t, \alpha \in[0,1]\}$, it can be deduced that

(i) $\widetilde{a} \leq \widetilde{b}$ if $a\left(t_{1}, \alpha\right) \leq b\left(t_{2}, \alpha\right), \forall t_{1}, t_{2}, \alpha \in[0,1]$,

(ii) $\widetilde{a} \unlhd_{w} \widetilde{b}$ if $a(t, \alpha) \leq b(t, \alpha), \forall t, \alpha \in[0,1]$.

It is easy to see that $\preceq$ and $\prec_{w}$ are partial order relations on $\mathscr{F}(\mathbb{R})$.

\section{Fuzzy-Valued Function and Its Differential Calculus}

A fuzzy-valued function is a function with fuzzy values, as $\tilde{f}: X \rightarrow \mathscr{F}(\mathbb{R})$, where $X$ is a subset of the vector space $\mathbb{R}^{n}$. Here, the fuzzy-valued functions with fuzzy coefficients are considered which allow us to express their $\alpha$-levels as a set of classical functions, using the parametric representation (2). To this end, let $\widetilde{\mathbf{C}}$ denote the set of all coefficients present in the fuzzy-valued function $\tilde{f}$, respectively. Without loss of generality, one can consider $\widetilde{\mathbf{C}}$ as an ordered set with respect to its presence in the fuzzy-valued function $\widetilde{f}$ (or as fuzzy vector like $\widetilde{\mathbf{C}}_{\gamma}^{k}$ ). Then, for a given fuzzy vector $\widetilde{\mathbf{C}}_{\nu}^{k}$, the $\alpha$ level of fuzzy-valued function $F_{\widetilde{\mathbf{C}}_{v}^{k}}: \mathbb{R}^{n} \rightarrow \mathscr{F}(\mathbb{R})$ can be considered as

$$
\begin{aligned}
& {\left[F_{\widetilde{\mathbf{C}}_{\nu}^{k}}(\mathbf{x})\right]^{\alpha}} \\
& \quad=\left\{f_{c(\mathbf{t}, \alpha)}(\mathbf{x}) \mid f_{c(\mathbf{t}, \alpha)}: \mathbb{R}^{n} \rightarrow \mathbb{R}, c(\mathbf{t}, \alpha) \in\left[\widetilde{\mathbf{C}}_{\nu}^{k}\right]^{\alpha}\right\} .
\end{aligned}
$$

For every fixed $\mathbf{x}$ and $\alpha \in[0,1], f_{c(\mathbf{t}, \alpha)}(\mathbf{x})$ is continuous in $\mathbf{t}$; consequently, $\min _{c(\mathbf{t}, \alpha) \in\left[\widetilde{\mathbf{C}}_{v}^{k}\right]^{\alpha}} f_{c(\mathbf{t}, \alpha)}(\mathbf{x})=\min _{\mathbf{t} \in[0,1]^{k}} f_{c(\mathbf{t}, \alpha)}(\mathbf{x})$ and $\max _{c(\mathbf{t}, \alpha) \in\left[\widetilde{\mathbf{C}}_{y}^{k}\right]^{\alpha}} f_{\mathcal{c}(\mathbf{t}, \alpha)}(\mathbf{x})=\max _{\mathbf{t} \in[0,1]^{k}} f_{c(\mathbf{t}, \alpha)}(\mathbf{x})$ exist and

$$
\left[F_{\widetilde{\mathbf{C}}_{v}^{k}}(\mathbf{x})\right]^{\alpha}=\left[\min _{\mathbf{t} \in[0,1]^{k}} f_{c(\mathbf{t}, \alpha)}(\mathbf{x}), \max _{\mathbf{t} \in[0,1]^{k}} f_{c(\mathbf{t}, \alpha)}(\mathbf{x})\right] .
$$

Example 6. Consider the fuzzy-valued function $F_{\widetilde{\mathbf{C}}^{3}}: \mathbb{R}^{2} \rightarrow$ $\mathscr{F}(\mathbb{R})$, where $F_{\widetilde{\mathbf{C}}_{v}^{3}}\left(x_{1}, x_{2}\right)=\widetilde{c}_{1} \odot x_{1}^{2} \oplus \widetilde{c}_{2} \odot \cos \left(\widetilde{\mathcal{c}}_{3} \odot x_{2}\right)$, $\widetilde{\mathrm{C}}_{\gamma}^{3}=\left(\widetilde{c}_{1}, \widetilde{c}_{2}, \widetilde{c}_{3}\right)=(\widetilde{5}, \widetilde{2}, \widetilde{3})$, and $\widetilde{5}=\langle 1,5,6\rangle, \tilde{2}=\langle 0,2,4\rangle$, and $\widetilde{3}=\langle 1,3,5\rangle$. For every $\alpha \in[0,1]$, by the parametric representation (3), we have

$$
\begin{aligned}
& {\left[\widetilde{\mathbf{C}}_{\nu}^{3}\right]^{\alpha}=\left\{c(\mathbf{t}, \alpha) \mid c(\mathbf{t}, \alpha)=\left(1+4 \alpha+t_{1}(5-5 \alpha), 2 \alpha\right.\right.} \\
& \left.\quad+t_{2}(4-4 \alpha), 1+2 \alpha+t_{3}(4-4 \alpha)\right)^{T}, \mathbf{t} \\
& \left.\quad=\left(t_{1}, t_{2}, t_{3}\right)^{T}, 0 \leq t_{i} \leq 1, \quad i=1,2,3\right\}
\end{aligned}
$$

and so

$$
\begin{aligned}
& {\left[F_{\widetilde{\mathbf{C}}_{\nu}^{3}}\right]^{\alpha}=\left\{f_{c(\mathbf{t}, \alpha)}\left(x_{1}, x_{2}\right) \mid f_{c(\mathbf{t}, \alpha)}\left(x_{1}, x_{2}\right)\right.} \\
& \quad=\left(1+4 \alpha+t_{1}(5-5 \alpha)\right) x_{1}^{2}+\left(2 \alpha+t_{2}(4-4 \alpha)\right) \\
& \quad \cdot \cos \left(\left(1+2 \alpha+t_{3}(4-4 \alpha)\right) x_{2}\right), \mathbf{t}=\left(t_{1}, t_{2}, t_{3}\right)^{T}, 0 \\
& \left.\quad \leq t_{i} \leq 1, i=1,2,3\right\} .
\end{aligned}
$$

Because of the continuity of $f_{c(\mathbf{t}, \alpha)}(x)$ at $\mathbf{t}$, for every $x$ and $\alpha \epsilon$ $[0,1],(9)$ provides that

$$
\begin{aligned}
& {\left[F_{\widetilde{\mathbf{C}}_{v}^{3}}\right]^{\alpha}=\left[(1+4 \alpha) x_{1}^{2}\right.} \\
& \quad+2 \alpha \cos \left((1+2 \alpha) x_{2}\right),(6-\alpha) x_{1}^{2} \\
& \left.\quad+(4-2 \alpha) \cos \left((5-2 \alpha) x_{2}\right)\right] .
\end{aligned}
$$

Definition 7. Let $F_{\widetilde{\mathbf{C}}_{\nu}^{k}}: \Omega \subseteq \mathbb{R}^{n} \rightarrow \mathscr{F}(\mathbb{R})$ be a fuzzy-valued function, where $\Omega$ is a convex subset of $\mathbb{R}^{n}$. $F_{\widetilde{\mathbf{C}}_{v}^{k}}$ is called convex on $\Omega$ with respect to $\preceq$ if

$$
F_{\widetilde{\mathbf{C}}_{v}^{k}}\left(\lambda \mathbf{x}_{1}+(1-\lambda) \mathbf{x}_{2}\right) \leq \lambda F_{\widetilde{\mathbf{C}}_{v}^{k}}\left(\mathbf{x}_{1}\right) \oplus(1-\lambda) F_{\widetilde{\mathbf{C}}_{v}^{k}}\left(\mathbf{x}_{2}\right),
$$

for all $\mathbf{x}_{1}, \mathbf{x}_{2} \in \Omega$ and $0 \leq \lambda \leq 1$. Moreover, the fuzzy-valued function $F_{\widetilde{\mathbf{C}}_{\nu}^{k}}$ is convex with respect to $\unlhd_{w}$, if (13) is valid for $\preceq_{w}$.

Remark 8. By Definitions 5 and 7, the convexity of fuzzyvalued function $F_{\widetilde{\mathbf{C}}_{v}^{k}}$ with respect to $\preceq$ or $\unlhd_{w}$ can be deduced by

$$
\begin{aligned}
& f_{c\left(\mathbf{t}^{\prime}, \alpha\right)}\left(\lambda \mathbf{x}_{1}+(1-\lambda) \mathbf{x}_{2}\right) \\
& \leq \lambda f_{c\left(\mathbf{t}^{\prime \prime}, \alpha\right)}\left(\mathbf{x}_{1}\right)+(1-\lambda) f_{c\left(\mathbf{t}^{\prime \prime}, \alpha\right)}\left(\mathbf{x}_{2}\right), \\
& \forall \alpha \in[0,1], \mathbf{t}^{\prime}, \mathbf{t}^{\prime \prime} \in[0,1]^{k},
\end{aligned}
$$




$$
\begin{aligned}
& f_{c(\mathbf{t}, \alpha)}\left(\lambda \mathbf{x}_{1}+(1-\lambda) \mathbf{x}_{2}\right) \\
& \quad \leq \lambda f_{c(\mathbf{t}, \alpha)}\left(\mathbf{x}_{1}\right)+(1-\lambda) f_{c(\mathbf{t}, \alpha)}\left(\mathbf{x}_{2}\right),
\end{aligned}
$$$$
\forall \alpha \in[0,1], \mathbf{t} \in[0,1]^{k},
$$

For any two arbitrary fuzzy vectors $\widetilde{\mathbf{C}}_{v}^{k}, \widetilde{\mathbf{D}}_{v}^{k} \in(\mathscr{F}(\mathbb{R}))^{k}$, the definition of algebraic operations between fuzzy-valued functions can be expressed, based on the parametric representation (8), as

respectively, for all $\mathbf{x}_{1}, \mathbf{x}_{2} \in \Omega$ and $0 \leq \lambda \leq 1$.

(i) $\left[F_{\widetilde{\mathbf{C}}_{v}^{k}}(\mathbf{x}) \circledast F_{\widetilde{\mathbf{C}}_{v}^{k}}(\mathbf{y})\right]^{\alpha}=\left\{f_{c(\mathbf{t}, \alpha)}(\mathbf{x}) * f_{c(\mathbf{t}, \alpha)}(\mathbf{y}) \mid f_{c(\mathbf{t}, \alpha)}: \mathbb{R}^{n} \longrightarrow \mathbb{R}, c(\mathbf{t}, \alpha) \in\left[\widetilde{\mathbf{C}}_{v}^{k}\right]^{\alpha}\right\}$,

(ii) $\left[F_{\widetilde{\mathbf{C}}_{v}^{k}}(\mathbf{x}) \circledast F_{\widetilde{\mathbf{D}}_{v}^{k}}(\mathbf{x})\right]^{\alpha}=\left\{f_{c(\mathbf{t}, \alpha)}(\mathbf{x}) * f_{d\left(\mathbf{t}^{\prime \prime}, \alpha\right)}(\mathbf{x}) \mid f_{c(\mathbf{t}, \alpha)}, f_{d\left(\mathbf{t}^{\prime \prime}, \alpha\right)}: \mathbb{R}^{n} \longrightarrow \mathbb{R}, c(\mathbf{t}, \alpha) \in\left[\widetilde{\mathbf{C}}_{v}^{k}\right]^{\alpha}, d\left(\mathbf{t}^{\prime \prime}, \alpha\right) \in\left[\widetilde{\mathbf{D}}_{v}^{k}\right]^{\alpha}\right\}$,

where $* \in\{+,-, \cdot, /\}$.

It is obvious that a metric to define the distance between two arbitrary fuzzy numbers is required to introduce the differential calculus of a fuzzy-valued function in the parametric form. Hereinafter, for two arbitrary fuzzy numbers $\widetilde{u}$ and $\widetilde{v}$, the quantity

$$
D(\widetilde{u}, \widetilde{v})=\sup _{0 \leq \alpha \leq 1}\left\{d\left([\tilde{u}]^{\alpha},[\widetilde{v}]^{\alpha}\right)\right\}
$$

describes the distance between $\widetilde{u}$ and $\widetilde{v}$, where

$$
\begin{aligned}
& d\left([\widetilde{u}]^{\alpha},[\widetilde{v}]^{\alpha}\right)=\max \left\{\max _{\mathbf{t}^{\prime}} \min _{\mathbf{t}^{\prime \prime}}\left|u\left(\mathbf{t}^{\prime}, \alpha\right)-v\left(\mathbf{t}^{\prime \prime}, \alpha\right)\right|,\right. \\
& \left.\max _{\mathbf{t}^{\prime \prime}} \min _{\mathbf{t}^{\prime}}\left|u\left(\mathbf{t}^{\prime}, \alpha\right)-v\left(\mathbf{t}^{\prime \prime}, \alpha\right)\right|\right\} .
\end{aligned}
$$

It is easy to see that the upper metric is equivalent to the wellknown Hausdorff metric [23].

Definition 9 (limit of fuzzy-valued function [24]). Let $F_{\widetilde{\mathbf{C}}_{\nu}^{k}}$ : $\mathbb{R}^{n} \rightarrow \mathscr{F}(\mathbb{R})$ be a fuzzy-valued function and a $\in \mathbb{R}^{n}, \widetilde{c} \in$ $\mathscr{F}(\mathbb{R})$. The limit of $F_{\widetilde{\mathbf{C}}_{\nu}^{k}}$ as $\mathbf{x}$ approaches $\mathbf{a}$ is the fuzzy number $\widetilde{c}$ and we write $\lim _{\mathbf{x} \rightarrow \mathbf{a}} F_{\widetilde{\mathbf{C}}_{v}^{k}}(\mathbf{x})=\widetilde{c}$, if for every $\varepsilon>0$ there exists $\delta>0$ such that $D\left(F_{\widetilde{\mathbf{C}}_{\nu}^{k}}(\mathbf{x}), \widetilde{\boldsymbol{c}}\right)<\varepsilon$, whenever $\|\mathbf{x}-\mathbf{a}\|<\delta$. Here, $\|\cdot\|$ is the usual (Euclidean) norm in $\mathbb{R}^{n}$.

Moreover, the fuzzy-valued function $F_{\widetilde{\mathbf{C}}_{\nu}^{k}}: \mathbb{R}^{n} \rightarrow \mathscr{F}(\mathbb{R})$ is continuous at $\mathbf{x}^{*} \in \mathbb{R}^{n}$ if and only if, for every $\varepsilon>0$, there exists $\delta=\delta\left(\mathbf{x}^{*}, \varepsilon\right)>0$ such that $\lim _{\mathbf{x} \rightarrow \mathbf{x}^{*}} F_{\widetilde{\mathbf{C}}_{v}^{k}}(\mathbf{x})=F_{\widetilde{\mathbf{C}}_{\nu}^{k}}\left(\mathbf{x}^{*}\right)$.

Proposition 10 (see [24]). The limit of fuzzy-valued function $F_{\widetilde{\mathbf{C}}_{v}^{k}}(\mathbf{x})$ exists at $\mathbf{x}^{*}$, if $\lim _{\mathbf{x} \rightarrow \mathbf{x}^{*}} f_{c(\mathbf{t}, \alpha)}(\mathbf{x})$ exists for every $c(\mathbf{t}, \alpha) \in$ $\left[\widetilde{\mathbf{C}}_{\gamma}^{k}\right]^{\alpha}$ and

$$
\begin{aligned}
\lim _{\mathbf{x} \rightarrow \mathbf{x}^{*}}\left[F_{\widetilde{\mathbf{C}}_{v}^{k}}(\mathbf{x})\right]^{\alpha}=\left[F_{\widetilde{\mathbf{C}}_{v}^{k}}\left(\mathbf{x}^{*}\right)\right]^{\alpha} \\
=\left[\min _{\mathbf{t}} \lim _{\mathbf{x} \rightarrow \mathbf{x}^{*}} f_{c(\mathbf{t}, \alpha)}(\mathbf{x}), \max _{\mathbf{t}} \lim _{\mathbf{x} \rightarrow \mathbf{x}^{*}} f_{c(\mathbf{t}, \alpha)}(\mathbf{x})\right] .
\end{aligned}
$$

Moreover, $F_{\widetilde{\mathbf{C}}_{\nu}^{k}}$ is continuous at $\mathbf{x}^{*}$ if and only if $f_{c(\mathbf{t}, \alpha)}$ is continuous at $\mathbf{x}^{*}$ for every $\mathbf{t} \in[0,1]^{k}$ and $\alpha \in[0,1]$.
One of the first definitions of differentiability for fuzzyvalued functions is the Hukuhara differentiability, which suffers disadvantages in particular to the point that the inverse subtraction does not exist. The generalized Hukuhara derivative has been attempted to clear these difficulties, which is more general than Hukuhara derivative. Finally, the generalized derivative is proposed based on the generalized difference [23]. Roughly speaking, all these derivatives vary with respect to their corresponding differences. Here, based on Definition 2, the following derivative can be defined in terms of parameter.

Definition 11 (differentiability of fuzzy-valued function). The fuzzy-valued function $F_{\widetilde{\mathbf{C}}_{\nu}^{k}}$ is said to be differentiable at $\mathbf{x}^{*}$, if $f_{c(\mathbf{t}, \alpha)}$ is differentiable at $\mathbf{x}^{*}$ for every $\mathbf{t} \in[0,1]^{k}$ and $\alpha \epsilon$ $[0,1]$ and $\left\{f_{c(\mathbf{t}, \alpha)}\left(\mathbf{x}^{*}+\mathbf{h}\right)-f_{c(\mathbf{t}, \alpha)}\left(\mathbf{x}^{*}\right), 0 \leq \alpha \leq 1\right\}$ satisfies the assumptions of Stacking Theorem [23].

In addition, the fuzzy-valued function $F_{\widetilde{\mathbf{C}}_{v}^{k}}$ is said to be differentiable on $\mathbb{R}^{n}$ if it is differentiable for all $\mathbf{x} \in \mathbb{R}^{n}$.

Proposition 12 (see [24]). If the fuzzy-valued function $F_{\widetilde{\mathbf{C}}_{\nu}^{k}}$ is differentiable at $\mathbf{x}^{*}$, then there exists a fuzzy number $F_{\widetilde{\mathrm{C}}_{v}^{k}}^{\prime}\left(\mathbf{x}^{*}\right)$ such that

$$
\begin{gathered}
{\left[F_{\widetilde{\mathrm{C}}_{v}^{k}}^{\prime}\left(\mathbf{x}^{*}\right)\right]^{\alpha}=\lim _{\mathbf{h} \rightarrow \mathbf{0}} \frac{\left[F_{\widetilde{\mathbf{C}}_{v}^{k}}\left(\mathbf{x}^{*}+\mathbf{h}\right) \ominus F_{\widetilde{\mathbf{C}}_{v}^{k}}\left(\mathbf{x}^{*}\right)\right]^{\alpha}}{\|\mathbf{h}\|}} \\
=\left[\min _{\mathbf{t} \in[0,1]^{k}} \lim _{\mathbf{h} \rightarrow \mathbf{0}} \frac{f_{c(\mathbf{t}, \alpha)}\left(\mathbf{x}^{*}+\mathbf{h}\right)-f_{c(\mathbf{t}, \alpha)}\left(\mathbf{x}^{*}\right)}{\|\mathbf{h}\|},\right. \\
\left.\max _{\mathbf{t} \in[0,1]^{k}} \lim _{\mathbf{h} \rightarrow \mathbf{0}} \frac{f_{c(\mathbf{t}, \alpha)}\left(\mathbf{x}^{*}+\mathbf{h}\right)-f_{c(\mathbf{t}, \alpha)}\left(\mathbf{x}^{*}\right)}{\|\mathbf{h}\|}\right] .
\end{gathered}
$$

Remark 13. Consider the fuzzy-valued function $F_{\widetilde{\mathbf{C}}_{v}^{2}}$ : $[-2,5] \rightarrow \mathscr{F}(R)$ defined as

$$
F_{\widetilde{\mathbf{C}}_{v}^{2}}(x)=-\widetilde{1} \odot x \oplus-\widetilde{2} \odot \sinh (x+2),
$$

where $-\tilde{1}=\langle-2,-1,3\rangle$ and $-\widetilde{2}=\langle-3,-2,-1\rangle$. Using gHderivative and Definition 11, we have 


$$
\begin{aligned}
& {\left[F_{\widetilde{\mathbf{C}}_{v}^{2}}^{\prime}(x)\right]^{\alpha}= \begin{cases}{[(3-4 \alpha)+(-3+\alpha) \cosh (x+2),(-2+\alpha)+(-1-\alpha) \cosh (x+2)],} & -2 \leq x \leq 0, \\
{[(-2+\alpha)+(-3+\alpha) \cosh (x+2),(3-4 \alpha)+(-1-\alpha) \cosh (x+2)],} & 0<x \leq 5,\end{cases} } \\
& {\left[F_{\widetilde{\mathbf{C}}_{v}^{2}}^{\prime}(x)\right]^{\alpha}=[(-2+\alpha)+(-3+\alpha) \cosh (x+2),(3-4 \alpha)+(-1-\alpha) \cosh (x+2)],}
\end{aligned}
$$

respectively, which are different. In fact, the sign of the independent variable $\mathbf{x}$ is not considered in Definition 11, while the gH-derivative depends on the sign of $\mathbf{x}$. Therefore, in general, it cannot be expected that the derivatives of a fuzzy-valued function be equal.

The partial derivative of $F_{\widetilde{\mathbf{C}}_{v}^{k}}$ in the direction $x_{i}$ at the point $\mathbf{x}^{*}$ can be defined in terms of its $\alpha$-level as

$$
\begin{gathered}
{\left[\frac{\partial F_{\widetilde{\mathbf{C}}_{\nu}^{k}}\left(\mathbf{x}^{*}\right)}{\partial x_{i}}\right]^{\alpha}=\left\{\frac{\partial f_{c(\mathbf{t}, \alpha)}\left(\mathbf{x}^{*}\right)}{\partial x_{i}} \mid \forall c(\mathbf{t}, \alpha) \in\left[\widetilde{\mathbf{C}}_{\nu}^{k}\right]^{\alpha}\right\}} \\
=\left[\min _{\mathbf{t} \in[0,1]^{k}} \frac{\partial f_{\mathcal{c}(\mathbf{t}, \alpha)}\left(\mathbf{x}^{*}\right)}{\partial x_{i}}, \max _{\mathbf{t} \in[0,1]^{k}} \frac{\partial f_{\mathcal{c}(\mathbf{t}, \alpha)}\left(\mathbf{x}^{*}\right)}{\partial x_{i}}\right],
\end{gathered}
$$

provided that, for every $\alpha \in[0,1], \partial f_{c(\mathbf{t}, \alpha)}\left(\mathbf{x}^{*}\right) / \partial x_{i}, i=$ $1,2, \ldots, n$, exist and they are the $\alpha$-level of a fuzzy number. Moreover, the gradient of fuzzy-valued function $F_{\widetilde{\mathbf{C}}_{y}^{k}}$ (i.e., the partial derivatives $\partial F_{\widetilde{\mathbf{C}}_{\nu}^{k}}\left(\mathbf{x}^{*}\right) / \partial x_{i}$ at the point $\left.\mathbf{x}^{*}\right)$ is defined as a fuzzy vector as follows:

$$
\begin{aligned}
& \nabla F_{\widetilde{\mathbf{C}}_{v}^{k}}\left(\mathbf{x}^{*}\right) \\
& \quad=\left(\frac{\partial F_{\widetilde{\mathbf{C}}_{v}^{k}}\left(\mathbf{x}^{*}\right)}{\partial x_{1}}, \frac{\partial F_{\widetilde{\mathbf{C}}_{v}^{k}}\left(\mathbf{x}^{*}\right)}{\partial x_{2}}, \ldots, \frac{\partial F_{\widetilde{\mathbf{C}}_{v}^{k}}\left(\mathbf{x}^{*}\right)}{\partial x_{n}}\right)^{T} .
\end{aligned}
$$

Definition 14. Suppose that the second-order partial derivatives $\partial^{2} f_{c(\mathbf{t}, \alpha)}\left(\mathbf{x}^{*}\right) / \partial x_{i} \partial x_{j}, i, j=1,2, \ldots, n$, at the point $\mathbf{x}^{*}$ exist and are $\alpha$-levels of fuzzy number (i.e., satisfying the assumption of Stacking Theorem). Then, the Hessian matrix of $F_{\widetilde{\mathbf{C}}_{v}^{k}}$ at the given point is given by

$$
\nabla^{2} F_{\widetilde{\mathbf{C}}_{v}^{k}}\left(\mathbf{x}^{*}\right)=\left(\frac{\partial^{2} F_{\widetilde{\mathbf{C}}_{v}^{k}}\left(\mathbf{x}^{*}\right)}{\partial x_{i} \partial x_{j}}\right)_{n \times n}, \quad \forall i, j=1,2, \ldots, n,
$$

where

$$
\begin{aligned}
& {\left[\frac{\partial^{2} F_{\widetilde{\mathbf{C}}_{v}^{k}}\left(\mathbf{x}^{*}\right)}{\partial x_{i} \partial x_{j}}\right]^{\alpha}} \\
& \quad=\left\{\frac{\partial^{2} f_{c(\mathbf{t}, \alpha)}\left(\mathbf{x}^{*}\right)}{\partial x_{i} \partial x_{j}} \mid \forall c(\mathbf{t}, \alpha) \in\left[\mathbf{C}_{\nu}^{k}\right]^{\alpha}\right\} \\
& \quad=\left[\min _{\mathbf{t} \in[0,1]^{k}} \frac{\partial^{2} f_{c(\mathbf{t}, \alpha)}\left(\mathbf{x}^{*}\right)}{\partial x_{i} \partial x_{j}}, \max _{\mathbf{t} \in[0,1]^{k}} \frac{\partial^{2} f_{c(\mathbf{t}, \alpha)}\left(\mathbf{x}^{*}\right)}{\partial x_{i} \partial x_{j}}\right], \\
& i, j=1,2, \ldots, n .
\end{aligned}
$$

It is apparent that

$$
\left[\nabla^{2} F_{\widetilde{\mathbf{C}}_{\nu}^{k}}\left(\mathbf{x}^{*}\right)\right]^{\alpha}=\left\{\nabla^{2} f_{c(\mathbf{t}, \alpha)}\left(\mathbf{x}^{*}\right) \mid \forall f_{c(\mathbf{t}, \alpha)} \in\left[F_{\widetilde{\mathbf{C}}_{\nu}^{k}}\right]^{\alpha}\right\} .
$$

Definition 15. The fuzzy-valued function $F_{\widetilde{\mathbf{C}}_{v}^{k}}$ is said to be twice continuously differentiable at $\mathbf{x}^{*}$, if its Hessian matrix at that point (i.e., $\nabla^{2} F_{\widetilde{\mathbf{C}}_{v}^{k}}\left(\mathbf{x}^{*}\right)$ ) exists and all of its components are continuous functions. Also, $F_{\widetilde{\mathbf{C}}_{y}^{k}}$ is twice continuously differentiable on $\mathbb{R}^{n}$, if it is twice continuously differentiable for all $\mathbf{x} \in \mathbb{R}^{n}$.

Furthermore, using Definition 14 and Proposition 10, it can be shown that the fuzzy-valued function $F_{\widetilde{\mathbf{C}}_{v}^{k}}$ inherits the twice continuous differentiability of $f_{c(\mathbf{t}, \alpha)}$ at $\mathbf{x}^{*}$, when $\partial^{2} f_{c(\mathbf{t}, \alpha)}\left(\mathbf{x}^{*}\right) / \partial x_{i} \partial x_{j}, i, j=1,2, \ldots, n$, are $\alpha$-levels of a fuzzy number.

Definition 16. A fuzzy matrix $\widetilde{\mathbf{A}}_{m}$ is said to be symmetric if each of its $\alpha$-levels is symmetric. Moreover, by the parametric representation (4), a fuzzy matrix $\widetilde{\mathbf{A}}_{m}$ is positive definite (or positive semidefinite) if every $A(\mathbf{t}, \alpha)$ is positive definite (or positive semidefinite).

Theorem 17 (see [24]). Let $F_{\widetilde{\mathbf{C}}_{\nu}^{k}}$ be a twice continuously differentiable fuzzy-valued function on the open convex set $\Omega \subseteq \mathbb{R}^{n}$. The function $F_{\widetilde{\mathbf{C}}_{v}^{k}}$ is convex with respect to $\unlhd_{w}$ if and only if its fuzzy Hessian matrix is positive semidefinite for all $\mathbf{x} \in \Omega$.

\section{Constrained Fuzzy-Valued Optimization Problem}

Consider the following constrained fuzzy-valued optimization problem:

$$
\begin{aligned}
\text { (CFOP) } \min & F_{\widetilde{\mathbf{C}}_{v}^{k}}(\mathbf{x}) \\
\text { s.t. } & G_{j \widetilde{\mathbf{D}}_{v}^{m_{j}}}(\mathbf{x}) \leq\left(\text { or } \unlhd_{w}\right) \widetilde{B}_{j} \\
& \\
&
\end{aligned}
$$

where $\widetilde{B}_{j} \in \mathscr{F}(\mathbb{R})$ for $j=1,2, \ldots, p$ and $F_{\widetilde{\mathbf{C}}_{v}^{k}}, G_{j \widetilde{\mathbf{D}}_{v}^{m_{j}}}$ : $\mathbb{R}^{n} \rightarrow \mathscr{F}(\mathbb{R})$ are fuzzy-valued functions with the parametric representations

$$
\begin{aligned}
& {\left[F_{\widetilde{\mathbf{C}}_{\nu}^{k}}(x)\right]^{\alpha}=\left\{f_{c(\mathbf{t}, \alpha)}(\mathbf{x}) \mid f_{c(\mathbf{t}, \alpha)}: \mathbb{R}^{n} \longrightarrow \mathbb{R}, c(\mathbf{t}, \alpha)\right.} \\
& \left.\quad \in\left[\widetilde{\mathbf{C}}_{\nu}^{k}\right]^{\alpha}\right\}
\end{aligned}
$$




$$
\begin{aligned}
& {\left[G_{j \widetilde{\mathbf{D}}_{v}^{m_{j}}}(\mathbf{x})\right]^{\alpha}=\left\{g_{j d\left(\mathbf{t}_{j}, \alpha\right)}(\mathbf{x}) \mid g_{j d\left(\mathbf{t}_{j}, \alpha\right)}(\mathbf{x}): \mathbb{R}^{n}\right.} \\
& \left.\quad \longrightarrow \mathbb{R}, d\left(\mathbf{t}_{j}, \alpha\right) \in\left[\widetilde{\mathbf{D}}_{v}^{m_{j}}\right]^{\alpha}\right\} .
\end{aligned}
$$

According to the partial orderings as discussed in Definition 5 and the parametric representations (3) and (4), the feasible region of the CFOP can be expressed as

$$
\begin{aligned}
& \mathbf{F}=\left\{x \in \mathbb{R}^{n}: G_{j \widetilde{\mathbf{D}}_{v}^{m_{j}}}(x) \preceq\left(\text { or } \unlhd_{w}\right) \widetilde{B}_{j} j=1,2, \ldots, p\right\}
\end{aligned}
$$

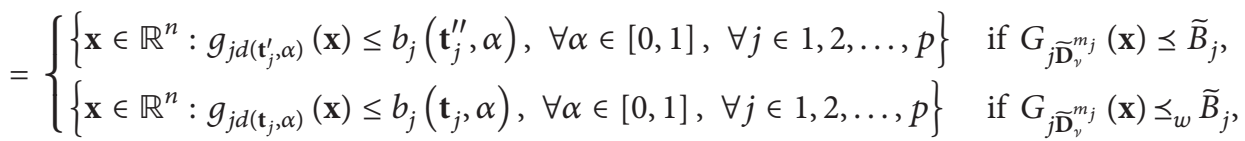

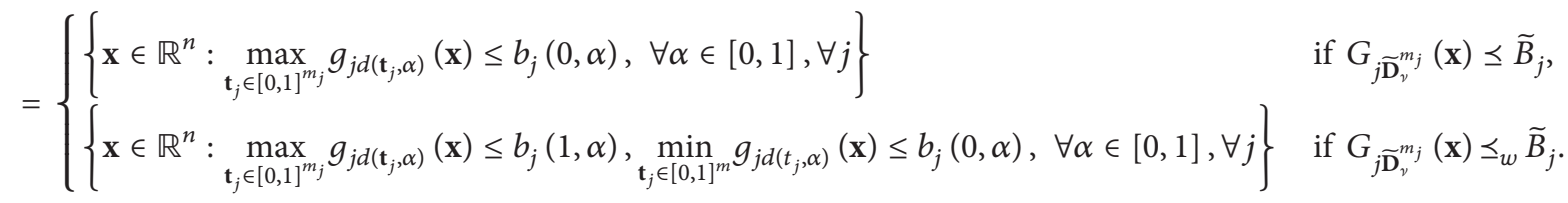

4.1. Solution Concepts and Optimality Conditions. In this section, a novel solution methodology was presented on the CFOP, which has depended on the definition of the corresponding optimal solution. Accordingly, it has been tried to define this concept based on the proposed partial orders. The feasible point $\mathbf{x}^{*}$ is said to be an optimal solution of the CFOP with respect to $\preceq_{w}$, if and only if

$$
F_{\widetilde{\mathbf{C}}_{v}^{k}}\left(\mathbf{x}^{*}\right) \preceq_{w} F_{\widetilde{\mathbf{C}}_{v}^{k}}(\mathbf{x})
$$

for all $\mathbf{x} \in \mathbf{F}$. Nevertheless, by the definition of the partial order $\preceq_{w}$, the CFOP can be handled via the following multiobjective problem:

$$
(\mathrm{COP})_{\mathbf{t}} \min _{\mathbf{x} \in \mathbf{F}} f_{c(\mathbf{t}, \alpha)}(\mathbf{x}) .
$$

So, the solution of the CFOP can be interpreted as the solution of $(\mathrm{COP})_{t}$, which is conforming to the concept of an efficient solution of a multiobjective problem. Consequently, the solution concept of the CFOP can be determined based on the thought of dominance.

Definition 18. Let $\mathbf{F} \subseteq \mathbb{R}^{n}$ be the feasible set of the CFOP

(i) A point $\mathbf{x}^{*} \in \mathbf{F}$ is an efficient solution of the CFOP if there is no $\mathbf{x} \in \mathbf{F}$, where

$$
\begin{aligned}
& f_{c(\mathbf{t}, \alpha)}(\mathbf{x}) \leq f_{c(\mathbf{t}, \alpha)}\left(\mathbf{x}^{*}\right) \\
& \forall \mathbf{t} \in[0,1]^{k}, F_{\widetilde{\mathbf{C}}_{v}^{k}}(\mathbf{x}) \neq F_{\widetilde{\mathbf{C}}_{v}^{k}}\left(\mathbf{x}^{*}\right) .
\end{aligned}
$$

(ii) A point $\mathbf{x}^{*} \in \mathbf{F}$ is said to be a properly efficient solution of the CFOP, if it is an efficient solution and there is a real number $\mu>0$ such that there exists at least one $\mathbf{t}^{\prime} \in[0,1]^{k}$, $\mathbf{t} \neq \mathbf{t}^{\prime}$, with $f_{c(\mathbf{t}, \alpha)}(\mathbf{x})>f_{c(\mathbf{t}, \alpha)}\left(\mathbf{x}^{*}\right)$, whereas

$$
\frac{f_{c(\mathbf{t}, \alpha)}\left(\mathbf{x}^{*}\right)-f_{c(\mathbf{t}, \alpha)}(\mathbf{x})}{f_{c(\mathbf{t}, \alpha)}(\mathbf{x})-f_{c(\mathbf{t}, \alpha)}\left(\mathbf{x}^{*}\right)} \leq \mu,
$$

for some $\mathbf{t} \in[0,1]^{k}$ and every $\mathbf{x} \in \mathbf{F}$ with $f_{c(\mathbf{t}, \alpha)}(\mathbf{x})<$ $f_{c(\mathbf{t}, \alpha)}\left(\mathbf{x}^{*}\right)$.
One of the main advantages of efficient solutions is to enable the decision maker to select one optimal solution that is matched best to his demand. In order to enhance the usefulness, the proposed solution concepts can be typically expanded as follows:

(1) CFOP has a weak efficient solution at $\mathbf{x}^{*} \in \mathbf{F}$, whenever relation (32) is established for some $\alpha \epsilon$ $[0,1]$.

(2) CFOP has a strong efficient solution at $\mathbf{x}^{*} \in \mathbf{F}$, if, for all $\alpha \in[0,1]$, relation (32) is valid.

(3) CFOP has a strong independent efficient solution at $\mathbf{x}^{*} \in \mathbf{F}$, when $\mathbf{x}^{*}$ is an efficient solution and it is independent of $\alpha$.

(4) CFOP has no efficient solution, if there is no $\mathbf{x}^{*} \in \mathbf{F}$ such that relation (32) is satisfied for any $\alpha \in[0,1]$.

Likewise, the concepts of weak, strong, strong independent, and no properly efficient solutions for the CFOP can be defined.

The fundamental idea to handle the considered multiobjective problem with infinity objective $(\mathrm{COP})_{\mathbf{t}}$ is to convert it to the following constrained single-objective optimization problem:

$$
\text { (COP) } \min _{\mathbf{x} \in \mathbf{F}} \int_{0}^{1} \int_{0}^{1} \cdots \int_{0}^{1} w(\mathbf{t}) f_{c(\mathbf{t}, \alpha)}(\mathbf{x}) d t_{1} d t_{2} \cdots d t_{k},
$$

where $w(\mathbf{t})=w\left(t_{1}, t_{2}, \ldots, t_{k}\right)$ is a weight function $w$ : $[0,1]^{k} \rightarrow(0,+\infty)$ and $t_{1}, t_{2}, \ldots, t_{k}$ are mutually independent. It can be shown that the solutions of the COP can be related to the CFOP ones.

Theorem 19. If $\mathbf{x}^{*} \in \mathbf{F}$ is an optimal solution of the COP, then it is a properly efficient solution of the CFOP.

Proof. By contradiction, assume that $\mathbf{x}^{*} \in \mathbf{F}$ is not a properly efficient solution of the CFOP. Therefore, $\mathbf{x}^{*}$ is not an efficient solution of the CFOP or the second part of Definition 18 
(ii) is violated. Anyway, for some $\mathbf{t} \in[0,1]^{k}$ and $\mathbf{x} \in \mathbf{F}$ with $f_{c(\mathbf{t}, \alpha)}(\mathbf{x})<f_{c(\mathbf{t}, \alpha)}\left(\mathbf{x}^{*}\right)$, pick out a continuous weight function $w:[0,1]^{k} \rightarrow(0,+\infty)$. Then, by choosing $\mu=$ $\max _{\left\{\mathbf{t} \neq \mathbf{t}^{\prime}, \mathbf{t}, \mathbf{t}^{\prime} \in[0,1]^{k}, w(\mathbf{t})>0\right\}}\left\{w\left(\mathbf{t}^{\prime}\right) / w(\mathbf{t})\right\}$, we have

$$
\frac{f_{c(\mathbf{t}, \alpha)}\left(\mathbf{x}^{*}\right)-f_{c(\mathbf{t}, \alpha)}(\mathbf{x})}{f_{c(\mathbf{t}, \alpha)}(\mathbf{x})-f_{c(\mathbf{t}, \alpha)}\left(\mathbf{x}^{*}\right)}>\mu
$$

for all $\mathbf{t}^{\prime} \in[0,1]^{k}$ with $f_{c(\mathbf{t}, \alpha)}(\mathbf{x})>f_{c(\mathbf{t}, \alpha)}\left(\mathbf{x}^{*}\right)$. Consequently,

$$
\begin{gathered}
f_{c(\mathbf{t}, \alpha)}\left(\mathbf{x}^{*}\right)-f_{c(\mathbf{t}, \alpha)}(\mathbf{x})>\mu\left(f_{c(\mathbf{t}, \alpha)}(\mathbf{x})-f_{c(\mathbf{t}, \alpha)}\left(\mathbf{x}^{*}\right)\right) \\
>\frac{w\left(\mathbf{t}^{\prime}\right)}{w(\mathbf{t})}\left(f_{c(\mathbf{t}, \alpha)}(\mathbf{x})-f_{c(\mathbf{t}, \alpha)}\left(\mathbf{x}^{*}\right)\right), \\
w(\mathbf{t}) f_{c(\mathbf{t}, \alpha)}\left(\mathbf{x}^{*}\right)-w(\mathbf{t}) f_{c(\mathbf{t}, \alpha)}(\mathbf{x}) \\
>w\left(\mathbf{t}^{\prime}\right) f_{c(\mathbf{t}, \alpha)}(\mathbf{x})-w\left(\mathbf{t}^{\prime}\right) f_{c(\mathbf{t}, \alpha)}\left(\mathbf{x}^{*}\right) .
\end{gathered}
$$

By integrating with respect to $t_{1}, t_{2}, \ldots, t_{k}$, we have

$$
\begin{gathered}
\int_{0}^{1} \int_{0}^{1} \cdots \int_{0}^{1} w(\mathbf{t}) f_{c(\mathbf{t}, \alpha)}\left(\mathbf{x}^{*}\right) d t_{1} d t_{2} \cdots d t_{k} \\
\quad-\int_{0}^{1} \int_{0}^{1} \cdots \int_{0}^{1} w(\mathbf{t}) f_{c(\mathbf{t}, \alpha)}(\mathbf{x}) d t_{1} d t_{2} \cdots d t_{k} \\
>\int_{0}^{1} \int_{0}^{1} \cdots \int_{0}^{1} w\left(\mathbf{t}^{\prime}\right) f_{c(\mathbf{t}, \alpha)}(\mathbf{x}) d t_{1}^{\prime} d t_{2}^{\prime} \cdots d t_{k}^{\prime} \\
\quad-\int_{0}^{1} \int_{0}^{1} \cdots \int_{0}^{1} w\left(\mathbf{t}^{\prime}\right) f_{c(\mathbf{t}, \alpha)}\left(\mathbf{x}^{*}\right) d t_{1}^{\prime} d t_{2}^{\prime} \cdots d t_{k}^{\prime} ;
\end{gathered}
$$

$$
\begin{aligned}
& \mathbf{F}=\left\{\mathbf{x} \in \mathbb{R}^{n}: \widetilde{\mathbf{A}}_{m} \mathbf{x} \preceq\left(\text { or } \preceq_{w}\right) \widetilde{\mathbf{B}}_{v}^{p}, \mathbf{x} \geq \mathbf{0}\right\} \\
& = \begin{cases}\left\{\mathbf{x} \in \mathbb{R}^{n}: A(1, \alpha) \mathbf{x} \leq b(0, \alpha), \mathbf{x} \geq \mathbf{0}, \forall j, \alpha\right\} & \text { if } \widetilde{\mathbf{A}}_{m} \mathbf{x} \leq \widetilde{\mathbf{B}}_{v}^{p}, \\
\left\{\mathbf{x} \in \mathbb{R}^{n}: A(1, \alpha) \mathbf{x} \leq b(1, \alpha), A(0, \alpha) \mathbf{x} \leq b(0, \alpha), \mathbf{x} \geq \mathbf{0}, \forall j, \alpha,\right\} & \text { if } \widetilde{\mathbf{A}}_{m} \mathbf{x} \unlhd_{w} \widetilde{\mathbf{B}}_{v}^{p} .\end{cases}
\end{aligned}
$$
solution of the COP. convex functions with respect to $\preceq_{w}$ or $\preceq$. convex programming problem.

Proof. It is the same as the proof of Theorem 3 of [25]. formulated as follows: $\mathrm{CFQP}$ can be obtained by the set

which contradicts the assumption that $\mathbf{x}^{*}$ is an optimal

It is noteworthy that a CFOP is said to be a constrained fuzzy-valued convex programming problem if $F_{\widetilde{\mathbf{C}}_{v}^{k}}, G_{j \widetilde{\mathbf{D}}_{v}{ }_{j}}$ are

Theorem 20. If the CFOP is a constrained fuzzy-valued convex programming problem, then the COP is a constrained

4.2. Constrained Fuzzy-Valued Quadratic Programming Problem. The constrained fuzzy-valued quadratic programming (CFQP) problem is a special case of the CFOP, when the fuzzy-valued function $F_{\widetilde{\mathbf{C}}_{v}^{k}}$ is quadratic and the constraints $G_{j} \widetilde{\mathbf{D}}_{v}^{m_{j}}$ are linear in $\mathbf{x} \in \mathbb{R}^{n}$. Generally, the problem can be

$$
\begin{aligned}
(\mathrm{CFQP}) \min & \widetilde{\mathbf{C}}_{v}^{n} \diamond \mathbf{x} \oplus \frac{1}{2} \odot \mathbf{x}^{T} \diamond \widetilde{\mathbf{Q}}_{m} \diamond \mathbf{x} \\
\text { s.t. } & \widetilde{\mathbf{A}}_{m} \mathbf{x} \preceq\left(\text { or } \unlhd_{w}\right) \widetilde{\mathbf{B}}_{v}^{p}, \quad \mathbf{x} \geq \mathbf{0}, \mathbf{x} \in \mathbb{R}^{n},
\end{aligned}
$$

where $\widetilde{\mathbf{Q}}_{m}=\left(\widetilde{\mathbf{Q}}_{i j}\right)_{n \times n} \in(\mathscr{F}(\mathbb{R}))^{n \times n}$ is a symmetric fuzzy matrix, $\widetilde{\mathbf{A}}_{m}=\left(\widetilde{\mathbf{A}}_{i j}\right)_{p \times n} \in(\mathscr{F}(\mathbb{R}))^{p \times n}, \widetilde{\mathbf{C}}_{v}^{n} \in(\mathscr{F}(\mathbb{R}))^{n}$, and $\widetilde{\mathbf{B}}_{v}^{p} \in(\mathscr{F}(\mathbb{R}))^{p}$. According to (29), the feasible region of the

It is self-evident that $\mathbf{F}$ is a convex set. Moreover, if the fuzzy Hessian matrix $\widetilde{\mathbf{Q}}_{m}$ is positive semidefinite then, by Theorem 17, the objective function will also be a fuzzy-valued convex function with respect to $\unlhd_{w}$. Consequently, the CFQP will be a convex optimization problem. On the other hand, its corresponding optimization problem with a weight function $w:[0,1]^{n^{2}+n} \rightarrow(0,+\infty)$ can be denoted by

$$
(\mathrm{CQP}) \min _{\mathbf{x} \in \mathbf{F}} \int_{0}^{1} \int_{0}^{1} \cdots \int_{0}^{1} w(\mathbf{t})\left\{c\left(\mathbf{t}^{\prime}, \alpha\right)^{T} \mathbf{x}+\frac{1}{2} \mathbf{x}^{T} Q\left(\mathbf{t}^{\prime \prime}, \alpha\right) \mathbf{x}\right\} d \mathbf{t}^{\prime} d \mathbf{t}^{\prime \prime}
$$

where $c\left(\mathbf{t}^{\prime}, \alpha\right) \in\left[\widetilde{\mathbf{C}}_{\nu}^{n}\right]^{\alpha}, Q\left(\mathbf{t}^{\prime \prime}, \alpha\right) \in\left[\widetilde{\mathbf{Q}}_{m}\right]^{\alpha}, d \mathbf{t}^{\prime}=$ $d t_{1}^{\prime} d t_{2}^{\prime} \cdots d t_{n}^{\prime}$, and $d \mathbf{t}^{\prime \prime}=d t_{i j}^{\prime \prime}, i, j=1,2, \ldots, m, \mathbf{t}=\left(\mathbf{t}^{\prime}, \mathbf{t}^{\prime \prime}\right)^{T}$.
By Theorem 20, the optimization problem (41) is also a constrained convex quadratic programming problem. Therefore, 
using KKT optimality conditions, its corresponding optimal solution can be obtained which is a properly efficient solution for (39).

Consider the feasible region $\mathbf{F}=\left\{\mathbf{x} \in \mathbb{R}^{n}: A(1, \alpha) \mathbf{x} \leq\right.$ $b(0, \alpha), \mathbf{x} \geq \mathbf{0}$; ; accordingly, the Lagrange function is obtained as

$$
\begin{aligned}
L(\mathbf{x}, \alpha, \lambda, \mu)= & h(\mathbf{x}, \alpha)+\lambda^{T}(A(1, \alpha) \mathbf{x}-b(0, \alpha)) \\
& -\mu^{T} \mathbf{x},
\end{aligned}
$$

where

$$
\begin{aligned}
& h(\mathbf{x}, \alpha)=\int_{0}^{1} \int_{0}^{1} \cdots \int_{0}^{1} w(\mathbf{t}) \\
& \cdot\left\{c\left(\mathbf{t}^{\prime}, \alpha\right)^{T} \mathbf{x}+\frac{1}{2} \mathbf{x}^{T} Q\left(\mathbf{t}^{\prime \prime}, \alpha\right) \mathbf{x}\right\} d \mathbf{t}^{\prime} d \mathbf{t}^{\prime \prime},
\end{aligned}
$$

and $\lambda \in \mathbb{R}^{p}, \mu \in \mathbb{R}^{n}, \lambda \geq 0$, and $\mu \geq 0$. So, the KKT optimality conditions are

$$
\begin{aligned}
& \nabla_{\mathbf{x}} L(\mathbf{x}, \alpha, \lambda, \mu) \\
& =\int_{0}^{1} \int_{0}^{1} \cdots \int_{0}^{1} w(t)\left\{c(\mathbf{t}, \alpha)^{T}+Q\left(t^{\prime \prime}, \alpha\right) \mathbf{x}\right\} \mathbf{d t}^{\prime} \mathbf{d t}^{\prime \prime} \\
& \quad+\lambda^{T} A(1, \alpha)=\mu^{T} \\
& \lambda^{T}(A(1, \alpha) \mathbf{x}-b(0, \alpha))=0 \\
& \mu^{T} \mathbf{x}=0
\end{aligned}
$$

where $\lambda \geq 0, \mu \geq 0$, and $\mathbf{x} \in \mathbf{F}$.

Furthermore, if the feasible region $\mathbf{F}$ takes another form in (40), then the KKT optimality conditions can be determined in a similar way.

\section{Numerical Examples}

In this section, two examples are given to illustrate the efficiency of the proposed approach. In the first example, the various solutions are discussed in detail and in the second one a special problem, namely, the constrained portfolio selection problem, is expressed.
Example 1. Consider the following constrained fuzzy-valued quadratic programming problem:

$$
\begin{array}{ll}
\min _{\mathbf{x} \in \mathbb{R}^{2}} & F_{\widetilde{\mathbf{C}}_{v}^{5}}\left(x_{1}, x_{2}\right) \\
= & \widetilde{6} \odot x_{1}^{2} \oplus \widetilde{4} \odot x_{1} x_{2} \oplus \widetilde{10} \odot x_{2}^{2} \oplus \widetilde{1} \odot x_{1} \oplus \widetilde{2} \\
& \odot x_{2} \\
\text { s.t. } \quad \widetilde{-2} \odot x_{1} \oplus \widetilde{-1} \odot x_{2} \preceq \widetilde{-1}, \\
& \widetilde{1} \odot x_{1} \oplus \widetilde{1} \odot x_{2} \preceq \widetilde{4}, \\
& x_{1}, x_{2} \geq 0,
\end{array}
$$

where $\tilde{6}=\langle 4,6,7\rangle, \tilde{4}=\langle 3,4,5\rangle, \widetilde{10}=\langle 9,10,12\rangle, \tilde{1}=$ $\langle 0,1,2\rangle, \widetilde{2}=\langle 0,2,4\rangle, \widetilde{-2}=\langle-4,-2,0\rangle$, and $\widetilde{-1}=\langle-2,-1,2\rangle$. The corresponding optimization problem with respect to $w$ : $[0,1]^{5} \rightarrow(0,+\infty)$ is

$$
\begin{aligned}
(\mathrm{CQP}) \min _{\mathbf{x} \in \mathbf{F}}{ }_{0}^{1} \int_{0}^{1} \cdots \int_{0}^{1} w(\mathbf{t}) \\
\cdot\left\{\left(4+2 \alpha+t_{1}(3-3 \alpha)\right) x_{1}^{2}\right. \\
+\left(3+\alpha+t_{2}(2-2 \alpha)\right) x_{1} x_{2} \\
+\left(9+\alpha+t_{3}(3-3 \alpha)\right) x_{2}^{2} \\
+\left(\alpha+t_{4}(2-2 \alpha)\right) x_{1} \\
\left.+\left(2 \alpha+t_{5}(4-4 \alpha)\right) x_{2}\right\} d \mathbf{t} \\
\mathbf{F}=\left\{\left(x_{1}, x_{2}\right):-2 \alpha x_{1}+(2-3 \alpha) x_{2}\right. \\
\leq-2+\alpha,(2-\alpha) x_{1}+(2-\alpha) x_{2} \\
\left.\leq 3+\alpha, x_{1}, x_{2} \geq 0\right\},
\end{aligned}
$$

where $d \mathbf{t}=d t_{1} d t_{2} d t_{3} d t_{4} d t_{5}$. The fuzzy-valued function $F_{\widetilde{\mathbf{C}}_{v}^{5}}$ is convex (see Example 2.2 [24]). Therefore, the constrained quadratic programming problem (46) is a convex programming problem, by Theorem 20. Accordingly, using the obtained result of Section 4.2, the KKT conditions for CQP are

$$
\begin{aligned}
& \int_{0}^{1} \int_{0}^{1} \cdots \int_{0}^{1} w(t)\left\{2\left(4+2 \alpha+t_{1}(3-3 \alpha)\right) x_{1}+\left(3+\alpha+t_{2}(2-2 \alpha)\right) x_{2}+\left(\alpha+t_{4}(2-2 \alpha)\right)\right\} \mathbf{d t}-2 \alpha \lambda_{1}+(2-\alpha) \lambda_{2} \\
& \quad=\mu_{1}, \\
& \int_{0}^{1} \int_{0}^{1} \cdots \int_{0}^{1} w(t)\left\{\left(3+\alpha+t_{2}(2-2 \alpha)\right) x_{1}+2\left(9+\alpha+t_{3}(3-3 \alpha)\right) x_{2}+\left(2 \alpha+t_{5}(4-4 \alpha)\right)\right\} \mathbf{d t}+(2-3 \alpha) \lambda_{1}+(2-\alpha) \\
& \quad \cdot \lambda_{2}=\mu_{2}, \\
& \lambda_{1}\left(-2 \alpha x_{1}+(2-3 \alpha) x_{2}-(-2+\alpha)\right)=0, \\
& \lambda_{2}\left((2-\alpha) x_{1}+(2-\alpha) x_{2}-(3+\alpha)\right)=0, \\
& \mu_{1} x_{1}=0, \mu_{2} x_{2}=0, \lambda_{1}, \lambda_{2}, \mu_{1}, \mu_{2} \geq 0,\left(x_{1}, x_{2}\right) \in \mathbf{F} .
\end{aligned}
$$


For a particular weight function $w(\mathbf{t})=t_{1}+t_{3}$, the above system can be simplified as

$$
\begin{aligned}
& \frac{23}{2} x_{1}+\frac{1}{2} x_{1} \alpha+4 x_{2}+1-2 \alpha \lambda_{1}+(2-\alpha) \lambda_{2}=\mu_{1}, \\
& 4 x_{1}+\frac{43}{2} x_{2}-\frac{3}{2} x_{2} \alpha+2+(2-3 \alpha) \lambda_{1}+(2-\alpha) \lambda_{2} \\
& \quad=\mu_{2}, \\
& \lambda_{1}\left(-2 \alpha x_{1}+(2-3 \alpha) x_{2}-(-2+\alpha)\right)=0, \\
& \lambda_{2}\left((2-\alpha) x_{1}+(2-\alpha) x_{2}-(3+\alpha)\right)=0, \\
& \mu_{1} x_{1}=0, \mu_{2} x_{2}=0, \lambda_{1}, \lambda_{2}, \mu_{1}, \mu_{2} \geq 0,\left(x_{1}, x_{2}\right) \in \mathbf{F} .
\end{aligned}
$$

For each $\alpha \in[0.3852,1]$, the solution of problem is $\left(x_{1}^{*}, x_{2}^{*}\right)=$ $(-0.5(\alpha-2) / \alpha, 0)$. Therefore, the fuzzy-valued optimization problem (45) has a weak properly efficient solution at $(-0.5(\alpha-2) / \alpha, 0)$.

Now, by considering some changes in the fuzzy-valued optimization problem (45), it can be shown that the solution of problem changes.

Case 1. Set $\widetilde{\mathbf{B}}^{\prime}{ }_{v}^{2}=[\widetilde{2} ; \widetilde{4}]$ instead of $\widetilde{\mathbf{B}}_{v}^{2}=[\widetilde{-1} ; \widetilde{4}]$. In this case, the following system may be obtained:

$$
\begin{aligned}
& \frac{23}{2} x_{1}+\frac{1}{2} x_{1} \alpha+4 x_{2}+1-2 \alpha \lambda_{1}+(2-\alpha) \lambda_{2}=\mu_{1}, \\
& 4 x_{1}+\frac{43}{2} x_{2}-\frac{3}{2} x_{2} \alpha+2+(2-3 \alpha) \lambda_{1}+(2-\alpha) \lambda_{2} \\
& \quad=\mu_{2}, \\
& \lambda_{1}\left(-2 \alpha x_{1}+(2-3 \alpha) x_{2}-2 \alpha\right)=0, \\
& \lambda_{2}\left((2-\alpha) x_{1}+(2-\alpha) x_{2}-(3+\alpha)\right)=0, \\
& -2 \alpha x_{1}+(2-3 \alpha) x_{2} \leq 2 \alpha, \\
& (2-\alpha) x_{1}+(2-\alpha) x_{2} \leq(3+\alpha), \\
& \mu_{1} x_{1}=0, \mu_{2} x_{2}=0, x_{1}, x_{2}, \lambda_{1}, \lambda_{2}, \mu_{1}, \mu_{2} \geq 0 .
\end{aligned}
$$

Consequently, the problem has a strong independent properly efficient solution at $\left(x_{1}^{*}, x_{2}^{*}\right)=(0,0)$.

Case 2. Set ${\widetilde{\mathbf{B}^{\prime}}}_{\gamma}^{2}=[\widetilde{-1} ; \widetilde{-4}]$ instead of $\widetilde{\mathbf{B}}_{v}^{2}=[\widetilde{-1} ; \widetilde{4}]$, where $\widetilde{-4}=\langle-5,-4,-2\rangle$ is a triangular fuzzy number; we have

$$
\begin{aligned}
& \frac{23}{2} x_{1}+\frac{1}{2} x_{1} \alpha+4 x_{2}+1-2 \alpha \lambda_{1}+(2-\alpha) \lambda_{2}=\mu_{1}, \\
& 4 x_{1}+\frac{43}{2} x_{2}-\frac{3}{2} x_{2} \alpha+2+(2-3 \alpha) \lambda_{1}+(2-\alpha) \lambda_{2} \\
& \quad=\mu_{2},
\end{aligned}
$$

$$
\begin{aligned}
& \lambda_{1}\left(-2 \alpha x_{1}+(2-3 \alpha) x_{2}-(-2+\alpha)\right)=0, \\
& \lambda_{2}\left((2-\alpha) x_{1}+(2-\alpha) x_{2}-(-5+\alpha)\right)=0, \\
& -2 \alpha x_{1}+(2-3 \alpha) x_{2} \leq-2+\alpha, \\
& (2-\alpha) x_{1}+(2-\alpha) x_{2} \leq-5+\alpha, \\
& \mu_{1} x_{1}=0, \mu_{2} x_{2}=0, x_{1}, x_{2}, \lambda_{1}, \lambda_{2}, \mu_{1}, \mu_{2} \geq 0,
\end{aligned}
$$

which has no solution, and so the problem has no properly efficient solution.

Case 3. Set $\widetilde{\mathbf{A}}_{m}^{\prime}=[\widetilde{-4}, \widetilde{2} ; \widetilde{-1}, \widetilde{-4}]$ instead of $\widetilde{\mathbf{A}}_{m}=$ $[\widetilde{-2}, \widetilde{-1} ; \widetilde{-1}, \widetilde{-1}]$. Therefore, the corresponding system is

$$
\begin{aligned}
& \frac{23}{2} x_{1}+\frac{1}{2} x_{1} \alpha+4 x_{2}+1+(-2-2 \alpha) \lambda_{1}+(2-3 \alpha) \lambda_{2} \\
& \quad=\mu_{1}, \\
& 4 x_{1}+\frac{43}{2} x_{2}-\frac{3}{2} x_{2} \alpha+2+(4-2 \alpha) \lambda_{1}+(2-3 \alpha) \lambda_{2} \\
& \quad=\mu_{2}, \\
& \lambda_{1}\left((-2-2 \alpha) x_{1}+(4-2 \alpha) x_{2}-(-2+\alpha)\right)=0, \\
& \lambda_{2}\left((2-3 \alpha) x_{1}+(2-3 \alpha) x_{2}-(3+\alpha)\right)=0, \\
& (-2-2 \alpha) x_{1}+(4-2 \alpha) x_{2} \leq-2+\alpha, \\
& (2-3 \alpha) x_{1}+(2-3 \alpha) x_{2} \leq 3+\alpha, \\
& \mu_{1} x_{1}=0, \mu_{2} x_{2}=0, x_{1}, x_{2}, \lambda_{1}, \lambda_{2}, \mu_{1}, \mu_{2} \geq 0,
\end{aligned}
$$

where, for each $\alpha \in[0,1]$, the pair $\left(x_{1}^{*}, x_{2}^{*}\right)=(-0.5(\alpha-2) /(\alpha+$ $1), 0)$ is the solution of the above system. Thus, the problem has a strong properly efficient solution at $(-0.5(\alpha-2) /(\alpha+$ 1),0).

To complete the discussion, it is interesting to explain the results by giving a special example named as portfolio selection problem. With respect to the mathematical programming problems including randomness and fuzziness, it is necessary to consider a certain optimization criterion so as to transform these problems into well-defined problems [26]. Therefore, in this paper, we consider fuzzy portfolio selection problem.

Example 2 (fuzzy portfolio selection problem [27]). The portfolio selection problem consists in selecting a portfolio of assets (or securities) that provides the investor with a given expected return and minimizes the risk. Mean-Variance optimization is probably the most popular approach to portfolio selection, which takes the variance of the portfolio as the measure of risk. It was introduced more than 50 years ago in the pioneering work by Markowitz [28].

Suppose that there are $n$ assets indexed by $i=1,2, \ldots, n$. Each asset $i$ is characterized by its random rate of return $r_{i}$, and its covariances with the rates of return of other assets are $\sigma_{i j}$ for $j=1,2, \ldots, n$. The matrix $\sigma_{n \times n}$ is symmetric and 
each diagonal element $\sigma_{i i}$ represents the variance of asset $i$. A positive value $R$ represents at least the desired rate of return.

The portfolio problem is to allocate total available wealth among these $n$ assets, allocating a fraction $x_{i}$ of wealth to the asset $i$. The value $\sum_{i, j=1}^{n} x_{i} \sigma_{i j} x_{j}$ represents the variance of the portfolio, and it is considered as the measure of the risk associated with the portfolio. Consequently, the problem is to minimize the overall variance, still ensuring the rate of return $R$. Thus, the problem can be formulated as follows:

$$
\begin{aligned}
\min _{x_{1}, x_{2}, \ldots, x_{n}} & \sum_{i, j=1}^{n} x_{i} \sigma_{i j} x_{j} \\
\text { s.t. } & \sum_{i=1}^{n} r_{i} x_{i} \geq R \\
& \sum_{i=1}^{n} x_{i}=1 .
\end{aligned}
$$

There may be the further restriction that each $x_{i} \geq 0$ which would imply that the assets must not be shorted. For $n=3$, we have

$$
\begin{array}{ll}
\min _{\mathbf{x} \in \mathbb{R}^{3}} & \frac{1}{2} \mathbf{x}^{T} Q_{m} \mathbf{x} \\
\text { s.t. } & -r_{1} x_{1}-r_{2} x_{2}-r_{3} x_{3} \leq-R \\
& x_{1}+x_{2}+x_{3}=1 \\
& \mathbf{x} \geq \mathbf{0},
\end{array}
$$

where

$$
\begin{aligned}
\mathbf{x} & =\left(x_{1}, x_{2}, x_{3}\right)^{T}, \\
Q_{m} & =\left(\begin{array}{ccc}
2 \sigma_{11} & \sigma_{12}+\sigma_{21} & \sigma_{13}+\sigma_{31} \\
\sigma_{12}+\sigma_{21} & 2 \sigma_{22} & \sigma_{23}+\sigma_{32} \\
\sigma_{13}+\sigma_{31} & \sigma_{23}+\sigma_{32} & 2 \sigma_{33}
\end{array}\right) .
\end{aligned}
$$

Since each asset is characterized by its random rate of return, then, for a closer look, we consider that the coefficients $\sigma_{i j}$, $r_{i}$, and $R$ become imprecise numbers. Thus, interpretation makes it flexible and allows us to have a class of solutions and also it helps us to improve the prediction and simulation and better assess the problem. In other words, the purpose is to introduce a model that, considering the uncertainty, the basket offers the best way to meet the demands of investors.

To have a typical application of this model, let the fuzzy optimization problem be of the following form:

$$
\begin{array}{ll}
\min _{\mathbf{x} \in \mathbb{R}^{3}} & \frac{1}{2} \odot \mathbf{x}^{T} \diamond \widetilde{\mathbf{Q}}_{m} \diamond \mathbf{x} \\
\text { s.t. } & -\widetilde{r}_{1} x_{1}-\widetilde{r}_{2} x_{2}-\widetilde{r}_{3} x_{3} \preceq-\widetilde{R} \\
& x_{1}+x_{2}+x_{3}=1 \\
& \mathbf{x} \geq \mathbf{0},
\end{array}
$$

where

$$
\begin{aligned}
\left(\widetilde{r}_{1}, \widetilde{r}_{2}, \widetilde{r}_{3}\right) & =(\widetilde{1}, \widetilde{3}, \widetilde{2}), \\
\widetilde{R} & =\widetilde{3}, \\
\widetilde{\mathbf{Q}}_{m} & =\left(\begin{array}{ccc}
\widetilde{6} & \widetilde{1} & \widetilde{4} \\
\widetilde{1} & \widetilde{9} & \widetilde{2} \\
\widetilde{4} & \widetilde{2} & \widetilde{16}
\end{array}\right),
\end{aligned}
$$

and $\tilde{1}=\langle 0,1,2\rangle, \tilde{2}=\langle 1,2,4\rangle, \tilde{3}=\langle 1,3,6\rangle, \tilde{4}=\langle 3,4,5\rangle$, $\widetilde{6}=\langle 4,6,9\rangle, \widetilde{9}=\langle 6,9,11\rangle$, and $\widetilde{16}=\langle 12,16,20\rangle$. One can easily check that $(1 / 2) \odot \mathbf{x}^{T} \diamond \widetilde{\mathbf{Q}}_{m} \diamond \mathbf{x}$ is a fuzzyvalued convex function from Definition 7 or Theorem 20. Using the method proposed in Section 4.2, the corresponding constrained convex quadratic programming, with the weight function $w:[0,1]^{6} \rightarrow(0,+\infty)$, is

$$
\begin{aligned}
& \min _{\mathbf{F}} \int_{0}^{1} \int_{0}^{1} \cdots \int_{0}^{1} \frac{1}{2} w(\mathbf{t}) \\
& \cdot\left\{\left(4+2 \alpha+t_{1}(5-5 \alpha)\right) x_{1}^{2}\right. \\
&+\left(6+3 \alpha+t_{2}(5-5 \alpha)\right) x_{2}^{2} \\
&+\left(12+4 \alpha+t_{3}(8-8 \alpha)\right) x_{3}^{2} \\
&+2\left(\alpha+t_{4}(2-2 \alpha)\right) x_{1} x_{2} \\
&+2\left(3+\alpha+t_{5}(2-2 \alpha)\right) x_{1} x_{3} \\
&\left.+2\left(1+\alpha+t_{6}(3-3 \alpha)\right) x_{2} x_{3}\right\} d \mathbf{t} \\
& \mathbf{F}=\left\{\left(x_{1}, x_{2}, x_{3}\right):-(2-\alpha) x_{1}-(6-3 \alpha) x_{2}\right. \\
&-(4-2 \alpha) x_{3} \leq-(1+2 \alpha), \\
&\left.x_{1}+x_{2}+x_{3}=1, x_{1}, x_{2}, x_{3} \geq 0\right\}
\end{aligned}
$$

where $\mathbf{d t}=d t_{1} d t_{2} \cdots d t_{6}$. For a particular weight function $w(\mathbf{t})=1$, one can obtain the following systems from KKT conditions:

$$
\begin{aligned}
& \frac{1}{2}\left(13 x_{1}+2 x_{2}+8 x_{3}-\alpha x_{1}\right)-(2-\alpha) \lambda_{1}+\lambda_{2}=\mu_{1}, \\
& \frac{1}{2}\left(2 x_{1}+17 x_{2}+5 x_{3}+\alpha x_{2}-\alpha x_{3}\right)-(6-3 \alpha) \lambda_{1} \\
& \quad+\lambda_{2}=\mu_{2}, \\
& \frac{1}{2}\left(8 x_{1}+5 x_{2}+32 x_{3}-\alpha x_{2}\right)-(4-2 \alpha) \lambda_{1}+\lambda_{2}=\mu_{3}, \\
& \lambda_{1}\left(-(2-\alpha) x_{1}-(6-3 \alpha) x_{2}-(4-2 \alpha) x_{3}\right. \\
& \quad+(1+2 \alpha))=0, \\
& \mu_{1} x_{1}=0, \mu_{2} x_{2}=0, \mu_{3} x_{3}=0, \lambda_{1}, \mu_{1}, \mu_{2}, \mu_{3} \geq 0, \\
& \quad\left(x_{1}, x_{2}, x_{3}\right) \in \mathrm{F} .
\end{aligned}
$$

For each $\alpha \in[0.9455,1]$, the solution of problem is $\left(x_{1}^{*}, x_{2}^{*}\right)=$ $(0,-(4 * \alpha-3) /(\alpha-2))$. Then, the problem has weak properly efficient solutions. 


\section{Conclusion}

This study identified that a specific parametric representation for the fuzzy number can clarify the fuzzy arithmetic and calculus of fuzzy-valued function which had several acceptable properties as flexibility, easy-to-control shapes, and applicability in practice. Furthermore, the various solution concepts associated with constrained fuzzy-valued optimization problem were outlined. More precisely, the constrained fuzzy-valued optimization problem with both fuzzy-valued objective function and constraints was converted to a general constrained optimization problem, based on its underlying fuzzy-valued functions. The ability of the proposed approach might help to consider more realistic modeling efforts in the real world, such as fuzzy portfolio selection problem as a prominent problem in the financial field.

\section{Conflicts of Interest}

The authors declare that there are no conflicts of interest regarding the publication of this paper.

\section{References}

[1] H. T. Nguyen, V. Kreinovich, B. Wu, and G. Xiang, Computing statistics under interval and fuzzy uncertainty, vol. 393 of Studies in Computational Intelligence, Springer, Berlin, Heidelberg, Germany, 2012.

[2] A. Sengupta and T. K. Pal, Fuzzy preference ordering of interval numbers in decision problems, vol. 238 of Studies in Fuzziness and Soft Computing, Springer, Berlin, Heidelberg, Germany, 2009.

[3] L. A. Zadeh, "Fuzzy sets," Information and Control, vol. 8, no. 3, pp. 338-353, 1965.

[4] H. R. Erfanian, M. J. Abdi, and S. Kahrizi, "An application of fuzzy linear programming in portfolio optimization," $M J$ Journal on Financial Mathematics, vol. 1, no. 1, pp. 1-10, 2016.

[5] N. S. Datta, H. S. Dutta, and K. Majumder, "Application of fuzzy logic and fuzzy optimization techniques in medical image processing," Biometrics: Concepts, Methodologies, Tools, and Applications, IGI Global, pp. 907-932, 2017.

[6] M. Verma and K. K. Shukla, "Application of fuzzy optimization to the orienteering problem," Advances in Fuzzy Systems, vol. 2015, Article ID 569248, 12 pages, 2015.

[7] S. Wang and S. Zhu, "On fuzzy portfolio selection problems," Fuzzy Optimization and Decision Making. A Journal of Modeling and Computation Under Uncertainty, vol. 1, no. 4, pp. 361-377, 2002.

[8] O. Abu Arqub, M. Al-Smadi, S. Momani, and T. Hayat, "Numerical solutions of fuzzy differential equations using reproducing kernel Hilbert space method," Soft Computing-A Fusion of Foundations, Methodologies and Applications, vol. 20, no. 8, pp. 3283-3302, 2016.

[9] O. Abu Arqub, S. Momani, S. Al-Mezel, and M. Kutbi, "Existence, uniqueness, and characterization theorems for nonlinear fuzzy integrodifferential equations of Volterra type," Mathematical Problems in Engineering, vol. 2015, Article ID 835891, 13 pages, 2015.

[10] O. A. Arqub, "Adaptation of reproducing kernel algorithm for solving fuzzy Fredholm-Volterra integrodifferential equations,"
Neural Computing and Applications, vol. 28, no. 7, pp. 1591-1610, 2017.

[11] O. A. Arqub, M. Al-Smadi, S. Momani, and T. Hayat, "Application of reproducing kernel algorithm for solving second-order, two-point fuzzy boundary value problems," Soft Computing, pp. $1-16,2016$.

[12] D. Ghosh, "A davidon-fletcher-powell type quasi-newton method to solve fuzzy optimization problems," in Proceedings of the International Conference on Mathematics and Computing ICMC 2017, vol. 655 of Communications in Computer and Information Science, pp. 232-245, Springer, Singapore, 2017.

[13] S. Tapaswini, S. Chakraverty, and T. Allahviranloo, "A new approach to $n$th order fuzzy differential equations," Computational Mathematics and Modeling, vol. 28, no. 2, pp. 278-300, 2017.

[14] Y. Zhang, X. Li, and S. Guo, "Portfolio selection problems with Markowitz's mean-variance framework: a review of literature," Fuzzy Optimization and Decision Making, pp. 1-34, 2017.

[15] Y. Chalco-Cano, W. A. Lodwick, and B. Bede, "Single level constraint interval arithmetic," Fuzzy Sets and Systems. An International Journal in Information Science and Engineering, vol. 257, pp. 146-168, 2014.

[16] R. E. Giachetti and R. E. Young, "A parametric representation of fuzzy numbers and their arithmetic operators," Fuzzy Sets and Systems. An International Journal in Information Science and Engineering, vol. 91, no. 2, pp. 185-202, 1997.

[17] L. Stefanini, L. Sorini, and M. L. Guerra, "Parametric representation of fuzzy numbers and application to fuzzy calculus," Fuzzy Sets and Systems. An International Journal in Information Science and Engineering, vol. 157, no. 18, pp. 2423-2455, 2006.

[18] B. Bede and S. G. Gal, "Generalizations of the differentiability of fuzzy-number-valued functions with applications to fuzzy differential equations," Fuzzy Sets and Systems. An International Journal in Information Science and Engineering, vol. 151, no. 3, pp. 581-599, 2005.

[19] S. Aytar, "Order intervals in the metric space of fuzzy numbers," Iranian Journal of Fuzzy Systems, vol. 12, no. 5, pp. 139-147, 2015.

[20] F. Molinari, "A new criterion of choice between generalized triangular fuzzy numbers," Fuzzy Sets and Systems. An International Journal in Information Science and Engineering, vol. 296, pp. 51-69, 2016.

[21] S. Nasseri, F. Taleshian, Z. Alizadeh, and J. Vahidi, "A new method for ordering lr fuzzy number," The Journal of Mathematics and Computer Science, vol. 4, no. 3, pp. 283-294, 2012.

[22] J. Ramík and J. ímánek, "Inequality relation between fuzzy numbers and its use in fuzzy optimization," Fuzzy Sets and Systems, vol. 16, no. 2, pp. 123-138, 1985.

[23] B. Bede and L. Stefanini, "Generalized differentiability of fuzzyvalued functions," Fuzzy Sets and Systems. An International Journal in Information Science and Engineering, vol. 230, pp. 119141, 2013.

[24] M. Heidari, M. R. Zadeh, O. S. Fard, and A. H. Borzabadi, "On unconstrained fuzzy-valued optimization problems," International Journal of Fuzzy Systems, vol. 18, no. 2, pp. 270-283, 2016.

[25] A. K. Bhurjee and G. Panda, "Efficient solution of interval optimization problem," Mathematical Methods of Operations Research, vol. 76, no. 3, pp. 273-288, 2012.

[26] T. Hasuike, H. Katagiri, and H. Ishii, "Portfolio selection problems with random fuzzy variable returns," Fuzzy Sets and Systems. An International Journal in Information Science and Engineering, vol. 160, no. 18, pp. 2579-2596, 2009. 
[27] A. Schaerf, "Local Search Techniques for Constrained Portfolio Selection Problems," Computational Economics, vol. 20, no. 3, pp. 177-190, 2002.

[28] H. Markowitz, "Portfolio selection," The Journal of Finance, vol. 7, no. 1, pp. 77-91, 1952. 


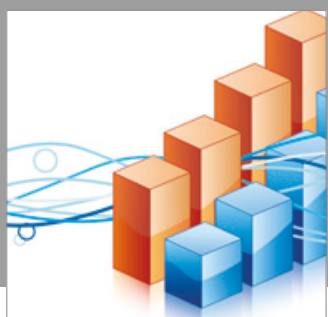

Advances in

Operations Research

vatersals

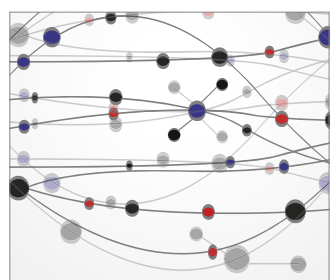

\section{The Scientific} World Journal
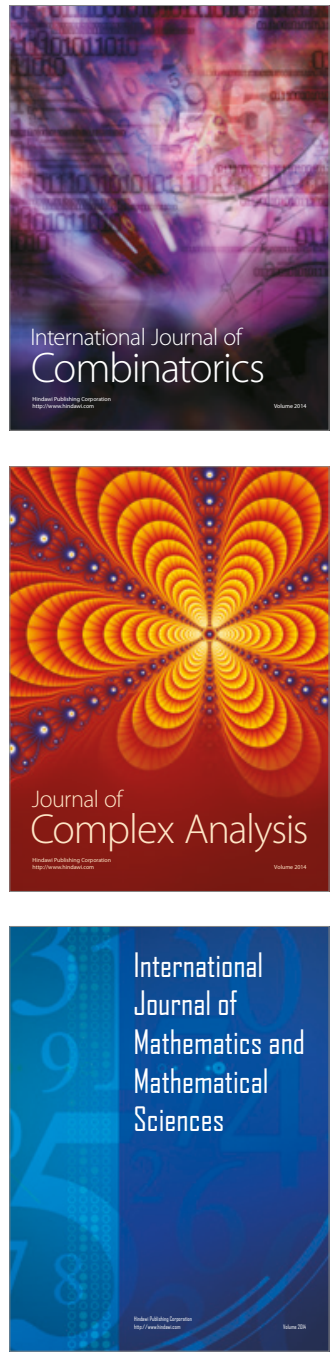
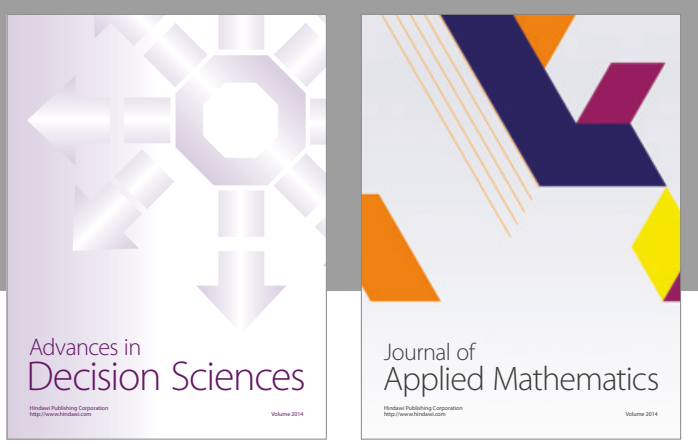

Algebra

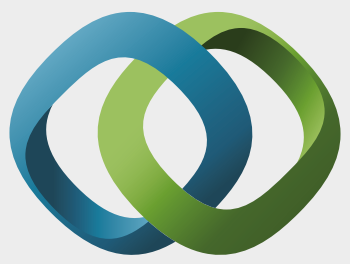

\section{Hindawi}

Submit your manuscripts at

https://www.hindawi.com
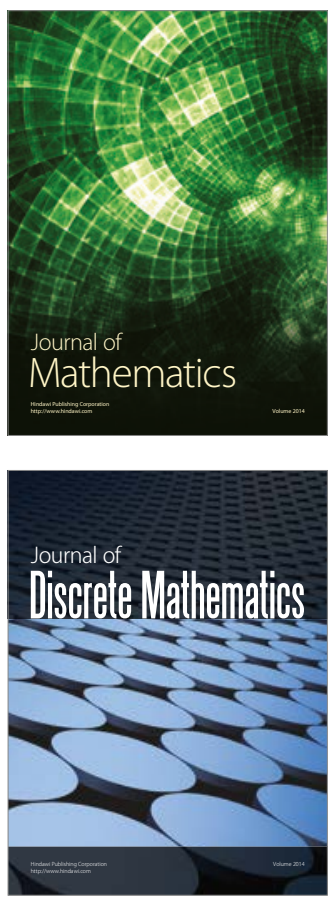

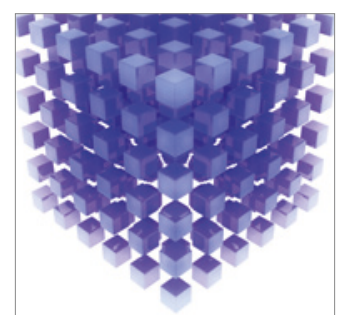

Mathematical Problems in Engineering
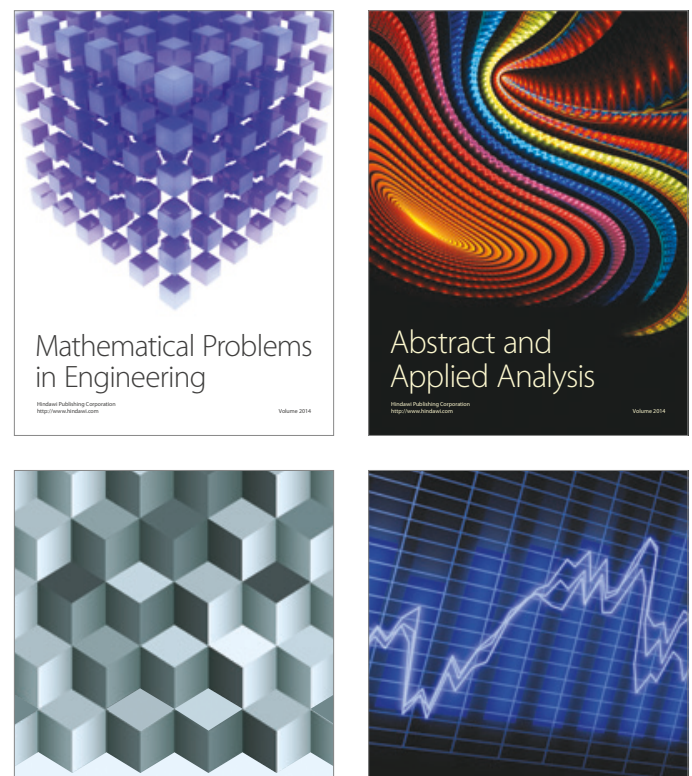

Journal of

Function Spaces

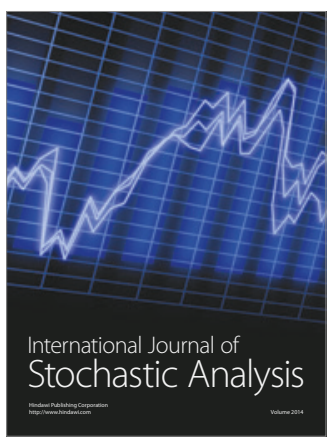

Probability and Statistics
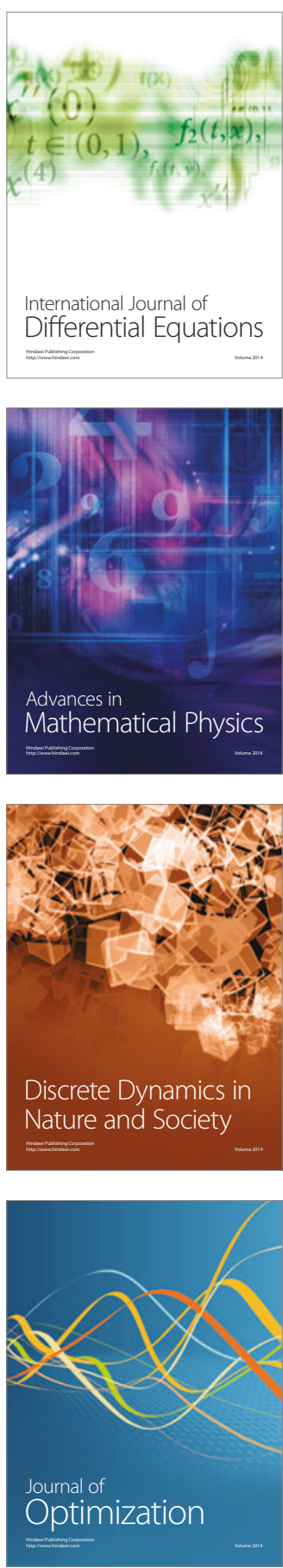